\title{
OPTIMIZATION OF MOBILE RADIOACTIVITY MONITORING NETWORKS
}

\author{
Z. Jiang $^{a} *$, S. de Bruin ${ }^{\text {a }}$, G.B.M. Heuvelink ${ }^{\mathrm{a}}$, C.J.W. Twenhöfel ${ }^{\mathrm{b}}$ \\ ${ }^{\text {a }}$ Wageningen University and Research Centre, P.O.Box 9101, 6700HB Wageningen, the Netherlands - \\ (Zhengkun.Jiang, Sytze.deBruin, Gerard.Heuvelink) @wur.nl \\ ${ }^{\mathrm{b}}$ National Institute for Public Health and the Environment (RIVM), P.O. Box 1, 3720 BA Bilthoven, the Netherlands - \\ Chris.Twenhofel@rivm.nl
}

KEY WORDS: optimization, semivariogram, spatial simulated annealing, monitoring networks

\begin{abstract}
:
In case of a nuclear accident, decision makers rely on high resolution and accurate information about the spatial distribution of the radioactivity levels in the surroundings of the accident site. Static nuclear monitoring networks are therefore employed in many countries in Europe. However, these networks were designed to cover the whole country and are usually too course to reach a high density in the local environment around the accident site. Therefore a strategy is considered in which the measurement density is increased during emergencies by adding measurements from mobile measuring devices. This raises the question where the mobile devices should be placed. This paper proposes a geostatistical methodology to optimize the allocation of the mobile devices, such that the expected weighed sum of false negative and false positive areas, i.e., false classification into safe and unsafe zones is minimized. The radioactivity concentration was modelled as the sum of a deterministic trend and a zero-mean spatially correlated stochastic residual, whereby the deterministic trend was defined as the outcome of a spatially explicit physical atmospheric dispersion model (NPK-PUFF model). The NPK-PUFF model used meteorological data and the characteristics of the radioactive release as input. The residual was characterized by a semivariogram that was estimated from the differences between outputs from various NPK-PUFF runs with input settings reflecting the uncertainty in the NPK-PUFF inputs (e.g., wind speed, wind direction). Spatial simulated annealing was used to obtain the optimal monitoring design, whereby accessibility and openness of sampling sites was also included. The method was computationally demanding but results were promising and the computational speed may be considerably improved to compute the optimal monitoring network in nearly real-time.
\end{abstract}

\section{INTRODUCTION}

\subsection{Background}

After the Chernobyl nuclear accident, most European governments increased efforts in operational systems for earlywarning and monitoring of radioactivity in the environment. In the Netherlands, the National Institute for Public Health and the Environment (RIVM) operates The Dutch National Radioactivity Monitoring Network (NRM). The RIVM constantly makes efforts to maintain and upgrade the network and has recently implemented its $3^{\text {rd }}$ generation network. The static monitoring network consists of 153 ambient dose rate monitors and $14 \alpha / \beta$ air sampling monitors (Twenhöfel et al., 2005).

The number and spatial distribution of the 153 ambient dose rate monitors is designed to cover the whole country. Prerequisite in any network design is that a significant release of radioactive material should be detected in time. Once the radiation level measurements are above a critical threshold, the NRM issues a warning to the national and local authorities. After confirmation and careful evaluation RIVM can activate the national emergency response plan for nuclear accidents (Twenhöfel et al., 2005). In a routine monitoring situation, measurements are carried out every 10 minutes by measuring devices at the static positions. From these measurements, NRM calculates hourly and daily averages and stores these in the database. In case of a large scale release of radioactivity in the environment, NRM plays an important role in the monitoring strategy during the response phase of a nuclear accident (Van Sonderen, 1997). In the early phase, the NRM provides realtime radiological information to support decisions on countermeasures. In the early stage the population is affected directly by radioactive contaminants via the exposure pathways of inhalation and external radiation. Protective countermeasures should therefore be carried out in the contaminated area where the radioactive plume passes and the dose levels may exceed intervention levels.

NRM measurements are converted into an effective dose using dispersion and dose projection models. Dispersion models are also used to predict the spatial distribution of radiation levels and make prognostic overviews of the radiological dose, integrated over a certain period of time, in the area surrounding the accident.

Since we are mainly concerned about momentary values of the radioactivity concentration in this study, we will evaluate possible areas of countermeasures in terms of action levels, i.e., deduced thresholds of momentary radioactivity concentrations

* corresponding author 
related to intervention levels by means of time integrated dose projections.

\subsection{Problem Definition}

The information derived from the static monitoring network and the dispersion model can be improved by adding measurements from mobile measuring devices. This requires a decision on how to optimally allocate the mobile measuring devices such that they provide maximum additional information. Here we assume that decision makers are mainly interested in minimizing the chance and costs of making wrong decisions on protective countermeasures. Therefore, the optimization goal is defined as minimizing the areas of false positives (i.e., the predicted concentration is greater than the action level while the real value is smaller) and false negatives (i.e., the predicted concentration is smaller than the action level while the real value is greater). In practice, a weighed combination of the false positives and false negatives may be used, since the costs associated with each of the two may be different.

Optimization of spatial sampling designs is an important research topic in geostatistics (Journel, 1988; Rouhani and Hall, 1988; Hudak and Loaiciga, 1992; Ridley et al., 1995). However, most of these studies adopt a simpler optimization criterion, namely to minimize the average kriging prediction error variance. Such a criterion is not helpful in our case of radioactivity monitoring and mapping, because uncertainty about the predicted radioactive concentration only affects decision making when the probability distribution of the prediction straddles the action level. Large uncertainties associated with predictions much below or much above the action level do no harm when it remains practically certain that the radioactivity is below or above the threshold. Therefore, existing geostatistical optimization methods need to be modified before used to address the problem in this work. Before presenting and applying a methodology for this, we first present the NPK-PUFF dispersion model and the geostatistical model that is used to characterize the spatial variability of radioactivity after a release.

\section{METHODOLOGY}

\subsection{NPK-PUFF Dispersion Model}

The RIVM and the Royal Dutch Meteorological Institute (KNMI) developed the NPK-PUFF dispersion model by adapting the PUFF model developed in the 1980's (Van Egmond and Kesseboom, 1983). The aim of the NPK-PUFF model is to calculate the concentration and deposition of radioactive materials that are released accidentally in the atmosphere. The NPK-PUFF model used in this work describes the atmospheric transport of gaseous and aerosol bounded radioactive nuclides from one or multiple point release sources up to distances of about $100 \mathrm{~km}$. The model output can be used to estimate (time-integrated) air and ground radioactivity concentration directly and consequently used in a dose estimation model to calculate the exposure of the population (Verver and De Leeuw, 1992).
NPK-PUFF uses hourly time steps and characterizes the mass distribution using Gaussian distributions. The mass is distributed over two vertical layers: a boundary layer and a reservoir layer. The path of the pollutant is determined either by analyzed or prognostic wind fields. These wind fields can be derived from data collected at meteorological stations or can be obtained from numerical weather prediction models. Every hour several meteorological parameters are updated by NPK-PUFF such as the boundary layer height, atmospheric stability and wind and rain field (Verver and De Leeuw, 1992).

\subsection{Geostatistical Model}

The dynamics of the radioactivity dispersion phenomenon in space and time can be reasonably well predicted with the NPKPUFF model, but model input and model structural error cause the model outputs to differ from the real phenomenon. Therefore, we propose a probabilistic model that treats the radiation as the sum of a deterministic trend (i.e., the NPKPUFF prediction) and a stochastic residual (Eq. (1)). It is assumed that the residual at any point in time is a normally distributed stationary random function with zero mean and variance and spatial correlation characterized by a semivariogram.

True concentration $(x, t)=N P K-P U F F(x, t)+\operatorname{residual}(x, t)(1)$

Thus, the distribution of the radioactive plume in space and time is largely explained by the NPK-PUFF physical dispersion model, which takes the influence of wind speed and direction and rainfall and other environmental factors into account. The underlying principles of assuming a spatially dependent residual are the First Law of Geography (Tobler, 1970) and Regionalized Variable (RV) theory (Matheron, 1971). The First Law of Geography states that "everything is related to everything else, but near things are more related than distant things". RV theory implements the first law of geography, by acknowledging that spatial correlation decreases with distance.

\subsection{Optimization Criterion}

As stated in the problem definition, the objective is to choose the mobile sampling locations such that the areas of false positives and false negatives are minimized. We now look into this issue in more detail and define the criterion to be minimized in mathematical forms. First, it should be noted that the areas of false positives and negatives change over time, meaning that some integrations over time would be needed to determine the optimal sampling scheme. Here, however, we have chosen to select a single time instant (typically several hours after the accident occurred) for which the criterion must be minimized. Secondly, we should also realise that since we have now assumed a stochastic model of reality, the incorrectly classified areas are uncertain, meaning that we must minimize the expected areas of false positives and negatives.

Given the action level, the predicted value at any location in the study area falls into any of four possible states: false positive, false negative, true positive and true negative (Fig. 1). The optimization criterion is to minimize a weighted sum of the expected area occupied by the two false states: 


$$
C(S)=E\left[\alpha \times A_{\text {false positive }}+\beta \times A_{\text {falsenegative }}\right]
$$

Here, E stands for mathematical expectation and $C(S)$ is the expected total cost using sampling design $S$, caused by false decisions; $\alpha$ and $\beta$ are the impact parameters of false positive prediction and false negative prediction respectively and A represents the area occupied. A differentiation between the cost associated with false positive and false negative areas is useful, because the associated costs are of a different nature and are unlikely to be equal (falsely assuming that an area is safe is presumably more 'costly' than falsely assuming that an area is unsafe, hence $\alpha<\beta$ ).

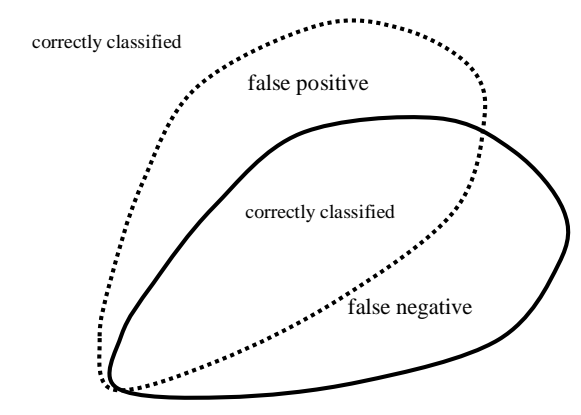

Figure 1. Graphical illustration of false positive and false negative areas. The Solid line delineates the 'true plume'; the dashed line delineates the 'predicted plume'.

The following step-by-step approach describes how the optimization objective is computed for a given sampling design (Fig. 2):

(1) Compute the NPK-PUFF output for the research area of interest;

(2) Define or estimate a semivariogram model of the stochastic residual;

(3) Generate a large number $(\mathrm{N} \geq 100)$ of realizations of the stochastic residual using unconditional sequential Gaussian simulation (Deutsch and Journel, 1998), and add these to the NPK-PUFF output, thus creating a set of $\mathrm{N}$ possible true concentration fields;

(4) For each of the $\mathrm{N}$ cases, compute the residual at the sampling locations and apply simple kriging to interpolate these into a map of the residual;

(5) For each of the $\mathrm{N}$ cases, add the map of the interpolated residual to the NPK-PUFF output to obtain a predicted concentration map;

(6) For each of the $\mathrm{N}$ cases, compare the predicted map with the 'true' concentration map; compute the associated area false positives and negatives and their weighted average;

(7) Compute the expected total costs by averaging the total costs over all simulations.

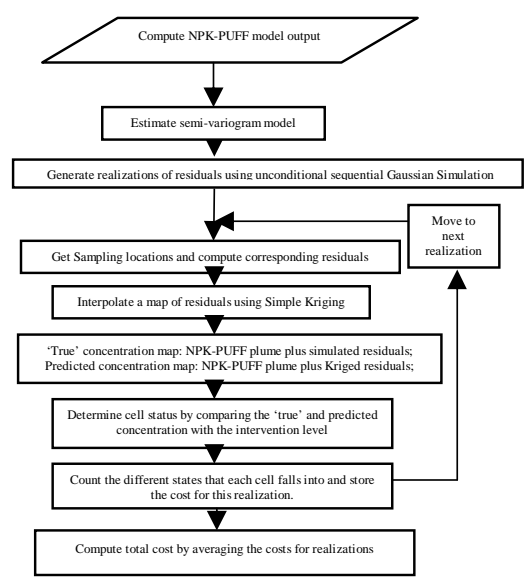

Figure 2. Flowchart of calculating the objective function

\subsection{Spatial Simulated Annealing}

Many algorithms have been developed for solving nonlinear optimization problems. Examples are as genetic algorithms, neural networks, tabu search and simulated annealing (Kunihiko, 1975; Schmitt, 2001; Glover, 1989). An empirical comparison of eight heuristic algorithms (Lee and Ellis,1996) showed that simulated annealing and tabu search exhibit superior performance. Simulated annealing (Kirkpatrick et al., 1983) is a random search technique which exploits an analogy with the physical process of annealing. The optimization problem is addressed iteratively by using a sequence of combinations, where each new combination is generated by deriving a new combination from slightly and randomly changing the previous combination. Spatial simulated annealing (SSA) is a special case of simulated annealing which deals with the optimization of a spatial sampling design (Van Groenigen et al., 1999).

The SSA steps are described as follows (see Van Groenigen et al. (1999) for details):

(1) Start with a (random) initial sampling scheme $S_{0}$ containing n sampling locations and compute objective function $C\left(S_{0}\right)$;

(2) Given the design $S_{k}$, construct a candidate new sampling design by moving a randomly chosen location over a distance $h$, whereby the direction of $h$ is random and its length is a random value between 0 and a maximum shift (the maximum shift is gradually decreased when the SSA iteration progresses);

(3) Compute the objective function for the new scheme. If the new scheme produced an improvement (smaller value for the cost function) then accept the new design as $S_{k+1}$. If the new design produces a worse result, then accept it with a certain probability smaller than one (the probability is gradually decreased as the SSA iteration progresses, towards the end it is close to zero);

(4) Return to step (2), using the new design $S_{k+1}$ as starting point if it was accepted and using the old design $S_{k}$ otherwise;

(5) Stop after a fixed number of iterations or using some other stopping criterion and store the design which had the smallest criterion value.

In practice, the optimization of a sampling scheme must also consider certain spatial constraints. For instance, potential sampling locations must satisfy openness and accessibility conditions. The former is important in order to avoid measurement artefacts (Twenhöfel et al., 2005). The latter is important, because installing the measurement devices by van 
must take place in a short time. These spatial constraints can be easily included by limiting the candidate sampling locations to a subset of the study area, namely the set of locations that satisfy the constraints.

\section{APPLICATION}

\subsection{Description of the case}

To demonstrate the proposed approach, we now present an example application following a hypothetical and, for the purpose of this work, greatly simplified accident, which is at time $t=0$ and at the nuclear power plant of Borssele in the Netherlands. A south-west wind (speed 3-4 ms ${ }^{-1}$ ) moves a plume of released Cs-137 in north-east direction. Our problem concerns optimal allocation of 25 mobile devices measuring ambient dose rates and whose measurements are used in combination with output from the NPK-PUFF dispersion model to assess the location and extent of the radioactive plume with a concentration above a certain action level at $\mathrm{t}=5$ hours.

The candidate sampling locations in our example are confined to a discrete set of accessible locations, i.e. we consider points on a $400 \mathrm{~m}$ square grid that are in open terrain and are within $300 \mathrm{~m}$ distance from the road network. The size of the study area is $100 \times 100 \mathrm{~km}^{2}$ and completely covers the plume five hours after the accident. (Fig. 3).

Rather than attempting to compute total costs $C(S)$ as defined in equation 3 , we use a relative cost in our optimization criterion. This relative cost is based on an assumed ratio between $\alpha$ (false positive impact parameter) and $\beta$ (false negative impact parameter) of $\alpha / \beta=1 / 5$. Accordingly, our optimization of sampling locations at $\mathrm{t}=5$ hours concerns minimization of the expected relative cost of the two erroneous states.

\subsection{NPK-PUFF model output}

Figure 3 shows the reference Cs- 137 plume for $t=5$ hours as predicted by the NPK-PUFF model. In addition 30 alternative NPK-PUFF outputs were produced by slightly changing some of the model parameters and model input values. The changes of model input values represent the expected uncertainty on the release parameters and meteorological conditions and are generally based on expert judgement (Kok, 2004). The model results were used only for parameterisation of the error model (see below).

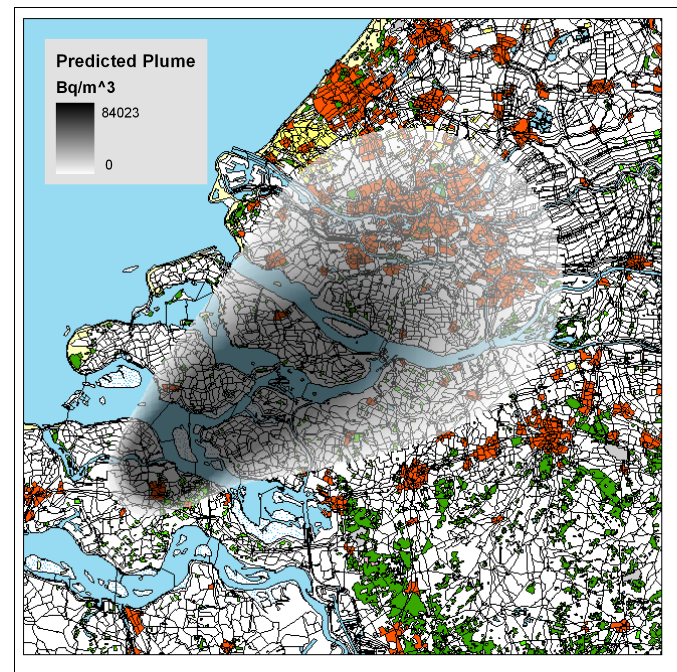

Figure 3. Plume predicted by the NPK-PUFF model, 5 hours after the hypothetical accident.

\subsection{Experimental semivariogram and model}

Thirty error fields were computed as the differences between the reference and the alternative NPK-PUFF model outputs. These error fields were randomly sampled and GSTAT (Pebsma and Wesseling, 1999) was used to compute 30 experimental semivariograms (Fig. 4). After observing these semivariograms, a spherical model (Eq. 3) was chosen to represent a scenario with medium variability of the differences between the NPKPUFF model output and reality and, hence, the measurements from the mobile devices.

$$
\gamma(h)=5.0 \times 10^{7} \operatorname{Sph}\left(3.0 \times 10^{4}\right)
$$

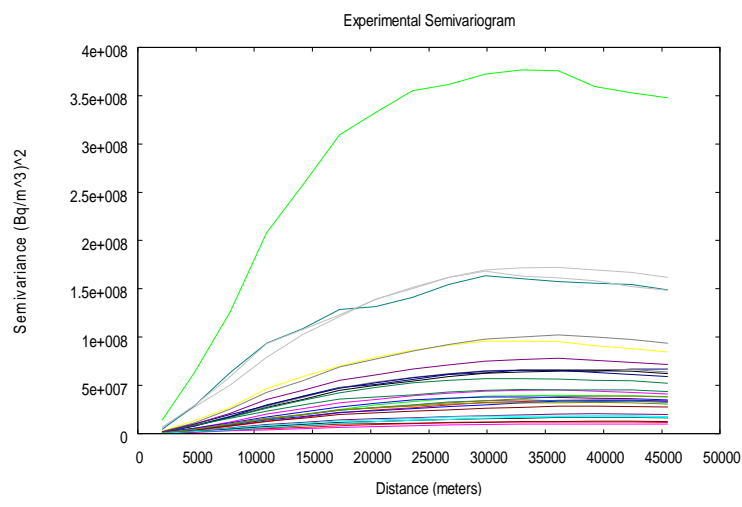

Figure 4. The 30 experimental semivariograms derived from 30 error fields.

\subsection{SSA optimization}

The example application was based on 100 simulated residual fields. Because of computational and time constraints, we stopped the SSA after 2000 steps. The initial probability of accepting a worsening design was set at 0.2 and it decreased exponentially as the SSA evolved. 
Figure 5 showed how the total cost associated with the sampling design decreased from 22264 for the initial design to 17942 for the final design, which is shown in Figure 6.

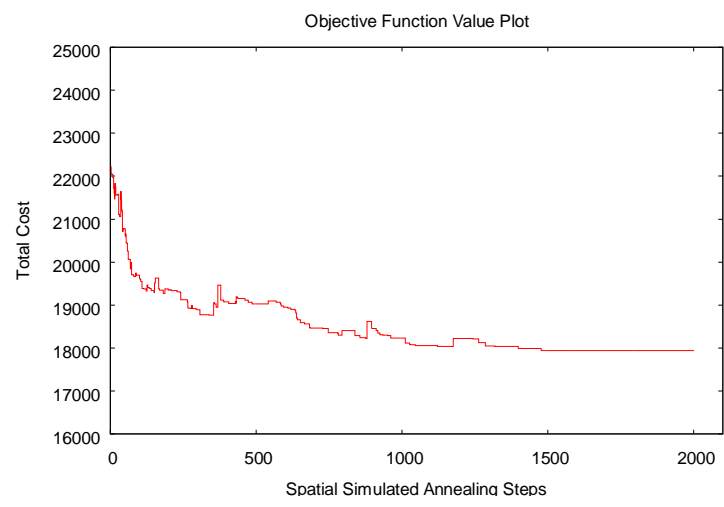

Figure 5. Cost criterion against spatial simulated annealing iteration steps

Figure 6 showed the initial sampling design and final sampling design respectively. The dots represented the mobile measuring devices, and they were selected from the discretized suitable area which meets the geographical constraints. The discretization was done by intersecting suitable area with the "fishnet" (a set of $1200 \mathrm{~m} * 1200 \mathrm{~m}$ square vectors that cover the whole study area). The solid line inside the plume delineated the threshold value of action level.

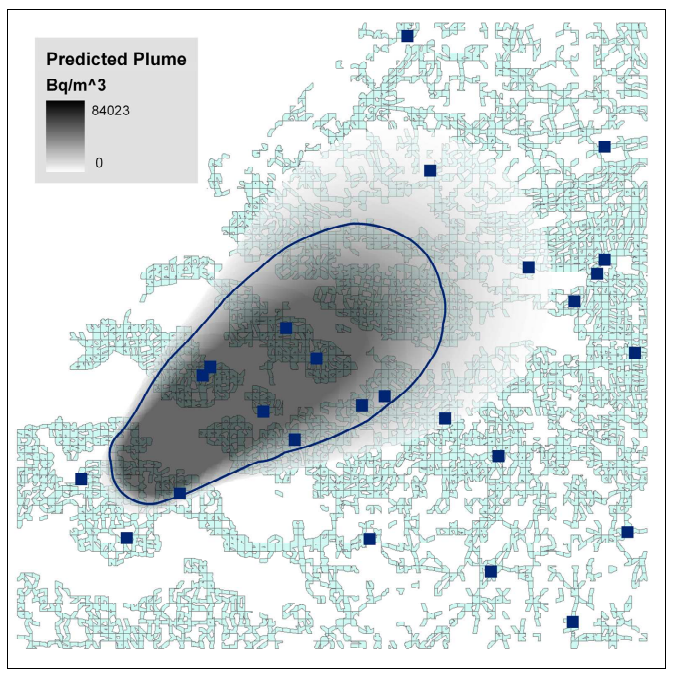

Initial sampling design

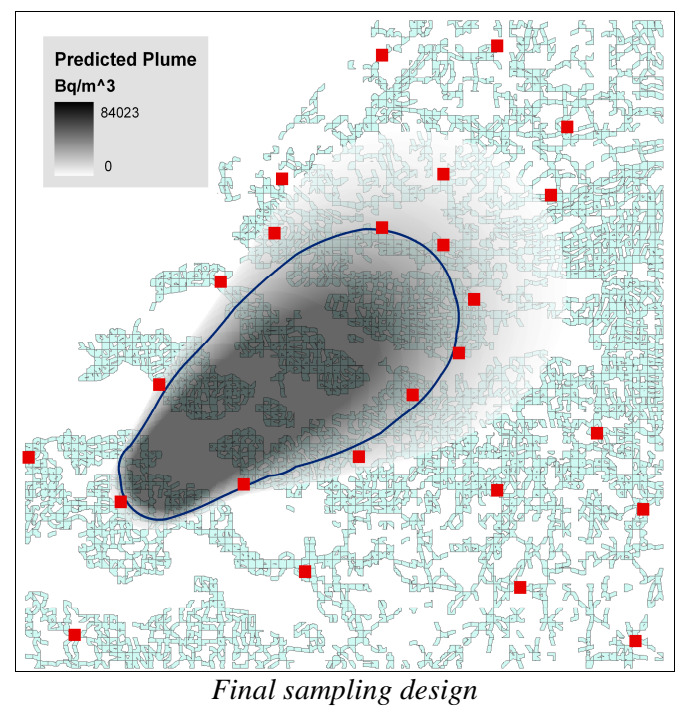

Figure 6. Initial and final sampling designs

As we can see from Fig. 6, the initial and randomly generated configuration was somewhat clustered and poorly covered the entire study area so that it resulted in a relatively high cost in objective function. For example, 10 points are clustered in the middle of the plume, which performed poorly in interpolation. In contrast, the final configuration selected by SSA algorithm presented a uniform coverage of study area. 13 observations were distributed along the plume internal border where became sensitive to threshold value by adding simulated or interpolated residuals and corresponded to the area of maximum uncertainty so that the observations effectively reduce the value of objective function. The rest of observations were spread over the area to control the variation brought by the limited 100 realizations.

Figures 7 and 8 showed the probabilities of false negative and positive predicted states under the initial and the final sampling design respectively.

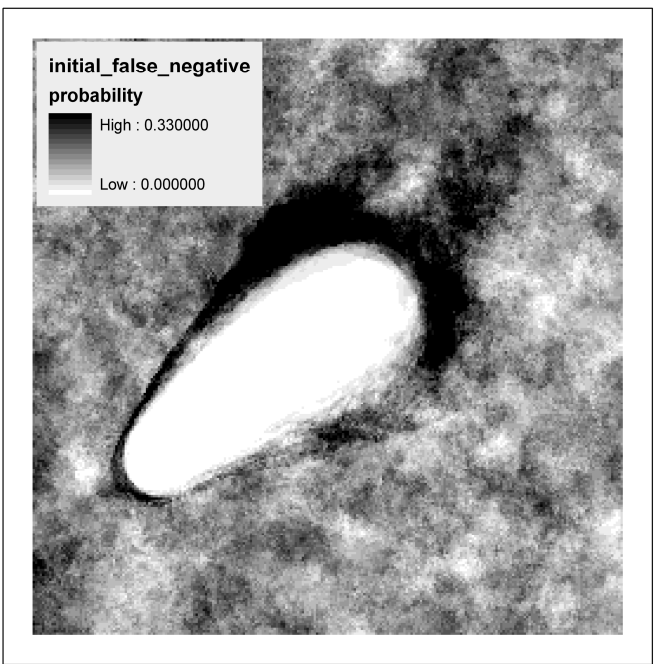

False negative area (initial) 


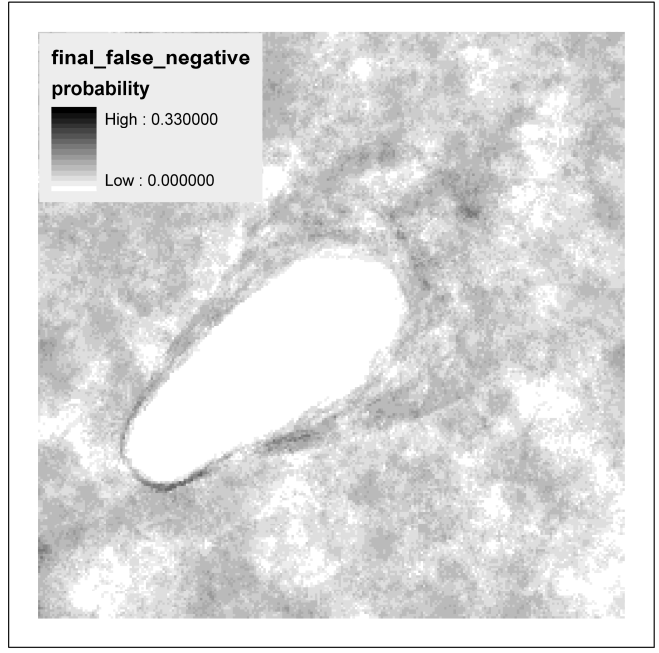

False negative area (final)

Figure 7. Probability maps of false negative areas

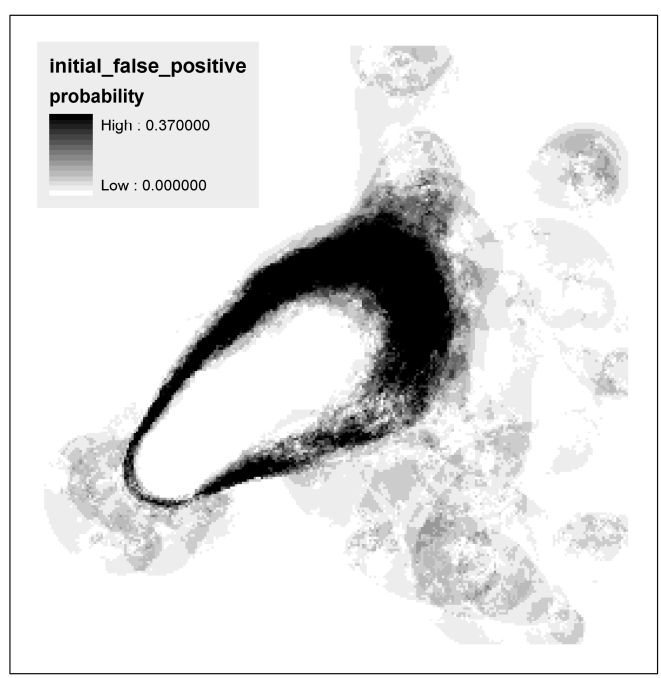

False positive area (initial)

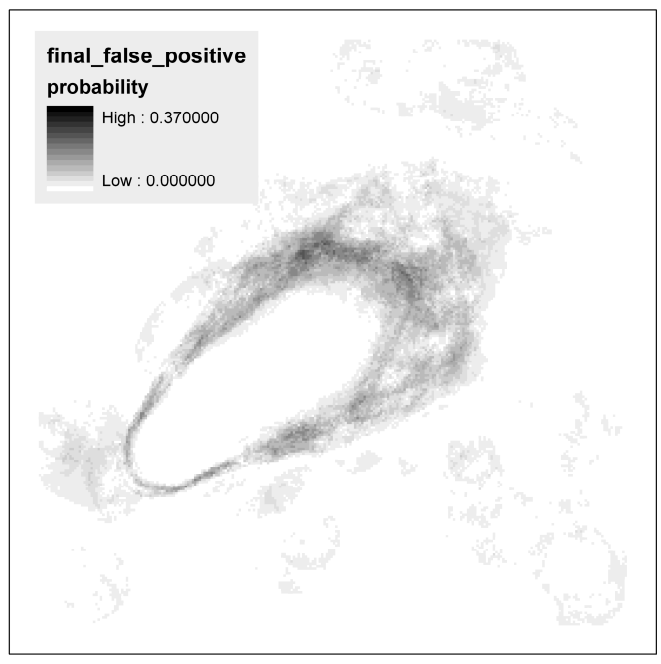

False positive area (final)

Figure 8. Probability maps of false positive areas
As we can see in Fig. 7 and Fig. 8, the probabilities of false prediction were decreased moderately. The maximum probability of false negative prediction was reduced from 0.33 to 0.20 and that of false positive prediction was reduced from 0.37 to 0.24 . The differences between initial with final probability maps accounted for the reason why certain location was selected. For instance, the probability of the lower right corner area decreased to 0 , after placed 5 additional observations.

Table 1 compared the situation that only the NPK-PUFF prediction was used with the one where NPK-PUFF prediction plus additional mobile measurements are used. It quantified the improvement after added mobile measuring devices.

\begin{tabular}{|c|c|c|c|}
\hline & $\begin{array}{c}\text { Expected } \\
\text { false } \\
\text { positive }\end{array}$ & $\begin{array}{c}\text { Expected } \\
\text { false } \\
\text { negative }\end{array}$ & Total cost \\
\hline $\begin{array}{c}\text { NPK-PUFF } \\
\text { prediction (without } \\
\text { observations) }\end{array}$ & 25725 & 978 & 26703 \\
\hline $\begin{array}{c}\text { NPK-PUFF } \\
\text { prediction }+ \\
\text { Mobile measuring } \\
\text { devices(final } \\
\text { design) }\end{array}$ & 16861 & 1081 & 17942 \\
\hline
\end{tabular}

Table 1 . The comparison showed the improvement brought by the additional mobile measuring devices.

Program implementation: the SSA procedure was coded and implemented in MATLAB (version: R2006b). On a Pentium IV computer with a CPU speed of $1.7 \mathrm{GHz}$ and $512 \mathrm{MB}$ RAM, it took 20 hours to finish 2000 SSA iterations.

\section{DISCUSSION AND CONCLUSIONS}

This study demonstrated that the objective to minimize the expected area of false predictions can be achieved by adopting a stochastic simulation approach and spatial simulated annealing. The computational effort is large, but results of the example application show that satisfactory results can be obtained with moderate effort. The objective function improved from 22264 to 17942 by replacing the randomly selected initial design with a final design that avoids clustering and concentrates observation points near the boundary of the NPK-PUFF plume. These initial results need to be further explored and analysis of how various model settings (semivariogram of stochastic residual, ratio of false negative and positive costs, time lag, number of mobile measurement stations) influence the resulting sampling design will provide valuable insight.

The results can be further improved by using more realizations and spatial simulated annealing iterations. Because only 100 realizations were incorporated, some random effects due to the limited number of simulated realities are visible in the resulting maps of false positives and negatives. Also, because of computational constraints we used only 2000 spatial simulated annealing iterations. We anticipate that the costs can be further reduced by increasing the number of iterations and fine tuning the simulated annealing parameters. 
We computed the predicted area of concentrations that are above the action level by adding the interpolated residual to the NPK-PUFF output and comparing at each location the result with the action level. However, when the costs of false negatives are much more expensive than those of false positives, it may be sensible to take a risk-aversive approach and classify a location positive when the probability of the concentration being above the action level is greater than a given number (e.g., 5, 10 or $25 \%$ ). This risk-aversive approach will decrease the expected area of false negatives, at the expense of an increased expected area of false positives. Overall, this may pay off when the costs of a false negative decision are greater than those of a false positive decision.

The geostatistical model used in this work assumes that the difference between the NPK-PUFF model output and reality is characterized with a zero-mean stationary stochastic residual. In future work, more elaborate representations of the error associated with the model output could be used. For instance, one possibility is to impose realistic uncertainties on the inputs to the NPK-PUFF model (e.g., wind speed and wind direction) and analyse how these propagate to uncertainty in the NPKPUFF output (Heuvelink, 1998).

In this paper, we have chosen one time instant for which the weighted expected area of false positives and negatives is minimized. An extension of the approach used would be to integrate the criterion over time so that a design is obtained which minimizes the expected false areas over a given time period. Integrating the criterion over time will extend the method to optimisation of the assessment of intervention levels instead of action levels. It is however somewhat more difficult because it will increase computing time and requires that the temporal correlation of the stochastic residual is modelled as well

\section{REFERENCES}

Deutsch, C.V., Journel, A.G., 1998. GS-LIB-Geostatistical Software Library and User's Guide. Oxford: University Press, Oxford.

Glover, F., 1989. Tabu search - part I. ORSA Journal of Computing, 1(3), pp: 190-206

Heuvelink, G.B.M., 1998. Error Propagation in Environmental Modelling with GIS. London: Taylor \& Francis, pp: 127- 132.

Hudak, P.F., Loaiciga, H.A., 1992. A location modelling approach for groundwater monitoring network augmentation. Water Resourse, 29(8), pp:2835-2845.

Journel, A.G., Huijbrechts, Ch.J., 1978. Mining geostatistics. Academic Press, London.

Journel, A.G., 1988. Nonparametric geostatistics for risk and additional sampling assessment. In: principal of environmental sampling, American Chemistry Society, Washington DC, pp:45-72.

Kirkpatrick, S., Gerlatt, C.D.Jr., Vecchi, M.P., 1983. Optimization by Simulated Annealing. Science, 220, pp: 671680
Kok, Y.S., Eleveld, H, Twenhöfel, C.J.W., 2004. Sensitivity and uncertainty analysis of the atmospheric dispersion model NPK-PUFF. In: Proceedings of the $9^{\text {th }}$ International Conference on Harmonisation within Atmospheric Dispersion Modelling for Regulatory Purposes, Garmisch Partenkirchen. Karlsruhe, Forschungszentrum Karlsruhe GmbH, pp: 79-83

Kunihiko, F, 1975. Cognitron: A self-organizing multilayered neural network. Biological Cybernetics, 20(3), pp: 121-136

Lee, Y.M., Ellis, J.H., 1996. Comparison of Algorithms for Nonlinear Integer Optimization: Application to Monitoring Network Design. Journal of environmental engineering, 122(6), pp: 524-531.

Matheron, G., 1971. The theory of regionalized variables, and their applications. Fasc. No.5, Cahiers du Centre de Morphologie Mathematique de Fontainebleau

Pebsma, E.J., Wesseling, C.G., 1998. Gstat: a program for geostatistical modelling, prediction and simulation. Computers \& Geosciences, 24(1), 17-31.

Ridley, M. N., Johnson, V. M., Tuckfield, R.C., 1995. Costeffective sampling of groundwater monitoring wells. Lawrence Livermore National Laboratory, Livermore, CA. UCRL-JC118909.

Rouhani, S., Hall, T.J., 1998. Geostatistical scheme for groundwater sampling. Journal of Hydrology. 103, pp: 85-120.

Schmitt, L. M., 2001. Theory of genetic algorithms. Theoretical Computer Science, 259(1-2), pp: 1- 61

Tobler, W. R., 1970. A computer model simulation of urban growth in the Detroit region. Economic Geography, 46(2), pp: 234-240.

Twenhöfel, C.J.W., van Beynen, C.de Hoog., van lunenburg, A.P.P.A., Slagt, G.J.E., Tax, R.B., van Westerlaak, P.J.M., Aldenkamp, F.J., 2005. Operation of the Dutch 3rd Generation National Radioactivity Monitoring Network. In: Spatial interpolation comparison 2004 report, EUR 21595 EN pp: 1934.

Van Egmond, N.D. and Kesseboom, H., 1983. Mesoscale air pollution dispersion models II; Lagrangian PUFF-model and comparison with eulerian GRID model. Atmospheric Environment, 17, pp. 265-274.

Van Groenigen, J.W., Siderius, W. Stein, A. 1999. Constrained optimization of soil sampling for minimization of the kriging variance. Geoderma 87, pp: 239-259.

Verver, G.H.L. and De Leeuw, F.A.A.M., 1992. An operational puff dispersion model. Atmospheric Environment, 26A(17), pp: 3179-3193.

Van Sonderen, J.F., 1997. Monitoring strategy in support of radiological emergency management. Radiation Protection Dosimetry, 73(1-4), pp: 115-118. 